\title{
EFFECTS OF ELECTRON DRIFTS ON THE COLLISIONLESS DAMPING OF KINETIC ALFVÉN WAVES IN THE SOLAR WIND
}

\author{
Yuguang Tong $^{1,2}$, Stuart D. Bale ${ }^{1,2}$, Christopher H. K. Chen ${ }^{1,3}$, Chadi S. Salem ${ }^{1}$, and Daniel Verscharen ${ }^{4}$ \\ ${ }^{1}$ Space Sciences Laboratory, University of California, Berkeley, CA 94720, USA; bale@ ssl.berkeley.edu \\ ${ }^{2}$ Department of Physics, University of California, Berkeley, CA 94720, USA; ygtong@ berkeley.edu \\ ${ }^{3}$ Department of Physics, Imperial College London, London SW7 2AZ, UK \\ ${ }^{4}$ Space Science Center, University of New Hampshire, Durham, NH 03824, USA \\ Received 2015 February 4; accepted 2015 April 16; published 2015 May 8
}

\begin{abstract}
The collisionless dissipation of anisotropic Alfvénic turbulence is a promising candidate to solve the solar wind heating problem. Extensive studies examined the kinetic properties of Alfvén waves in simple Maxwellian or biMaxwellian plasmas. However, the observed electron velocity distribution functions in the solar wind are more complex. In this study, we analyze the properties of kinetic Alfvén waves (KAWs) in a plasma with two drifting electron populations. We numerically solve the linearized Maxwell-Vlasov equations and find that the damping rate and the proton-electron energy partition for KAWs are significantly modified in such plasmas, compared to plasmas without electron drifts. We suggest that electron drift is an important factor to take into account when considering the dissipation of Alfvénic turbulence in the solar wind or other $\beta \sim 1$ astrophysical plasmas.
\end{abstract}

Key words: acceleration of particles - plasmas - solar wind - turbulence - waves

\section{INTRODUCTION}

One major question in space physics is how collisionless plasmas in the extended corona and in the solar wind are heated. Observations in the solar wind reveal that the fluctuations of the electric and magnetic fields show a turbulent spectrum similar to the power-law spectrum of fluid turbulence as described by Kolmogorov (Tu \& Marsch 1995; Bale et al. 2005; Alexandrova et al. 2013; Bruno \& Carbone 2013). The turbulence in the solar wind shows mainly Alfvénic polarization (Belcher \& Davis 1971) and becomes more anisotropic during the cascade to higher wavenumbers $k$ (Goldreich \& Sridhar 1995; Horbury et al. 2008; Chen et al. 2010), resulting in $k_{\perp} \gg k_{\|}$at short wavelengths. At the proton scale, the turbulence is thought to transition into a kinetic Alfvén wave (KAW) cascade (Schekochihin et al. 2009; Sahraoui et al. 2010; Howes et al. 2011; Boldyrev \& Perez 2012; Salem et al. 2012; Chen et al. 2013), which gradually dissipates energy to the particles (Leamon et al. 1999; Howes et al. 2011).

Despite the nonlinear nature of turbulence, linear theory has been used in studies of solar wind turbulence (see, for instance, Howes et al. 2014 for review). These studies analyze turbulent fluctuations by means of the linear propagation and damping characteristics of KAWs to help to understand the dissipation of the turbulence and the heating of the solar wind. The vast majority of these studies rely on representing the velocity distribution functions (VDFs) by an isotropic or bi-Maxwellian background (e.g., Quataert 1998; Leamon et al. 1999; Cranmer \& van Ballegooijen 2003). In this work, we investigate how more realistic VDFs modify the damping of KAWs. In the solar wind, both ions and electrons can be modeled by several populations drifting with respect to each other. Recent studies show that the differential flow between ions affects ion heating by both ion-cyclotron waves (Kasper et al. 2013) and lowfrequency KAWs (Chandran et al. 2013).

The solar wind electron VDF can be modeled as a superposition of three electron populations: a cool and dense "core," a hot and less dense "halo" (Feldman et al. 1975) and a beam-like one-sided "strahl" (Maksimovic et al. 2005). In the proton frame, core (halo and strahl) electrons drift sunward (anti-sunward) along the background magnetic field. Empirically, the solar wind fulfills quasi-neutrality and the zerocurrent condition (Feldman et al. 1975; Pulupa et al. 2014). The uncertainty in the fitting parameters for electron core properties is smaller than the uncertainty in the fitting parameters for halo and strahl properties. A drifting biMaxwellian population models core electrons very well. In particular, the core electron bulk drift speed is usually comparable to or larger than the Alfvén speed and shows a clear statistical dependence on collisional age (Pulupa et al. 2014). In the extrapolated asymptotic limit of no collisions, the core drift is as large as three to four times the Alfvén speed.

In this letter, we examine how the drift between electron core and halo affects the linear damping of KAWs. We model the solar wind electrons by a superposition of a Maxwellian core and a Maxwellian halo drifting against each other in the proton frame. Choosing a set of parameters typical for the solar wind, we solve the full hot-plasma dispersion relation of KAWs in the framework of Vlasov-Maxwell theory and identify the contribution to wave damping from each plasma component. We show that in the linear approximation, electron drifts can lead to significant variations in both the KAW damping rates and the relative energy transfer from the waves to protons and electrons, the latter of which is another important unsolved problem in turbulent plasma heating.

\section{THEORY AND METHOD}

Motivated by observed solar wind electron VDFs, we consider a collisionless, homogeneous, and warm protonelectron plasma in a constant uniform background magnetic field. The proton temperature and density are denoted as $T_{p}$ and $n_{p}$; the electron distribution consists of two shifted Maxwellian populations, a cool $\left(T_{c}\right)$ "core" and a hot $\left(T_{h}\right)$ "halo." As an initial step to study the effects of core electron drifts on resonant damping, we use isotropic temperatures for all plasma 


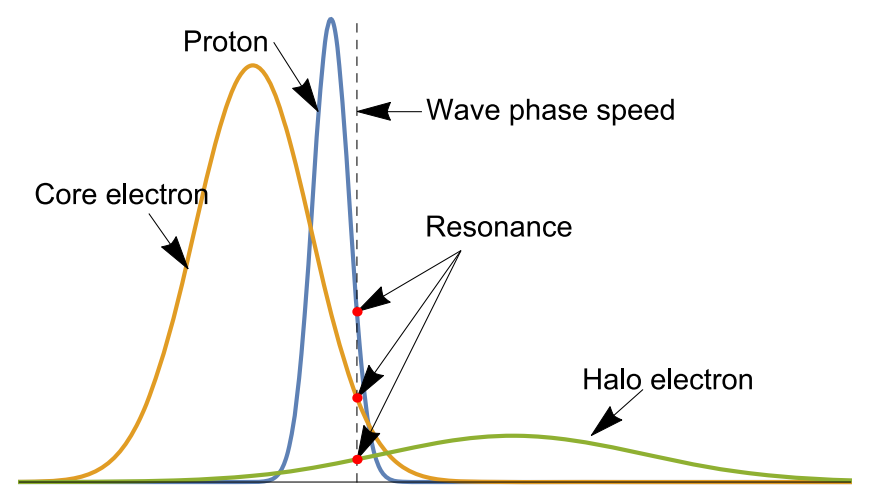

Figure 1. Schematic diagram for the plasma considered in this letter. We annotate the parallel wave phase speed $v_{\text {res }}=\omega_{r} / k_{\|}$for our resonance analysis in Section 3.

components. Both electron populations drift along the background magnetic field $\boldsymbol{B}_{0}=B_{0} \hat{\mathbf{z}}$ with $\boldsymbol{v}_{c}=v_{c} \hat{\mathbf{z}}$ and $\boldsymbol{v}_{h}=v_{h} \hat{\mathbf{z}}$ in the proton frame. The halo electron drift adjusts to the core electron drift to guarantee the absence of net currents in the plasma: $v_{h}=-v_{c} n_{c} / n_{h}$, where $n_{c}$ and $n_{h}$ denote core and halo electron densities. Figure 1 shows the plasma described above schematically.

Under the assumption of strong anisotropy in kinetic scale fluctuations in the solar wind, we consider KAWs with wavevectors at a large angle with respect to the magnetic field. We use constant values for the angle between $\boldsymbol{k}$ (wavenumber vector) and $\boldsymbol{B}_{0}\left(89^{\circ}\right.$ or $\left.91^{\circ}\right)$, plasma beta, the electron-core-to-proton temperature ratio and the core-to-halo density ratio, so that the only free parameters are $\boldsymbol{k}, \boldsymbol{v}_{c}$ and $\operatorname{sign}\left(\boldsymbol{k} \cdot \boldsymbol{B}_{0}\right)$. In particular, we allow $\boldsymbol{B}_{0}$ to be either parallel or anti-parallel to $\boldsymbol{v}_{c}$, and $\boldsymbol{k}$ has a finite component along $\boldsymbol{B}_{0}$. In principle, each configuration $\left(\boldsymbol{v}_{c}, \boldsymbol{k}, \boldsymbol{B}_{0}\right)$ requires a separate treatment; however, changing the direction of $\boldsymbol{B}_{0}$ only inverts the direction of propagation and does not produce a new dispersion relation. Therefore, we introduce the dimensionless core electron drift $\delta v_{c} \equiv\left|v_{c} / v_{\mathrm{A}}\right| \operatorname{sign}\left(\boldsymbol{k} \cdot \boldsymbol{v}_{c}\right)$, where $v_{\mathrm{A}} \equiv \sqrt{B_{0}^{2} / 4 \pi n_{p} m_{p}}$ is the proton Alfvén speed. The values of the remaining parameters are given in Table 1 .

We study the dispersion relations of KAWs by numerically solving the full set of the linear Maxwell-Vlasov equations. Linear Maxwell-Vlasov theory has been described in detail in the literature (see, for instance, Swanson 1989 and Stix 1992). We summarize the relevant results here. Linear plasma waves are eigenmodes of the wave equation:

$$
\boldsymbol{k} \times(\boldsymbol{k} \times \boldsymbol{E})+\frac{\omega^{2}}{c^{2}} \boldsymbol{\epsilon} \cdot \boldsymbol{E}=0,
$$

where $\boldsymbol{E}$ is the fluctuating electric field in Fourier space. The dielectric tensor $\epsilon$ incorporates contributions from each plasma component: $\epsilon=\mathbf{1}+\Sigma_{s} \chi_{s}$, where $\chi_{s}$ is the susceptibility tensor for species $s$. We obtain $\chi_{s}$ from the linearized and Fouriertransformed Vlasov equation and Maxwell's equations.

To study the contribution to wave damping (growth) from each component, we calculate the relative particle heating rates as (Stix 1992)

$$
\left.P_{s} \equiv \boldsymbol{E}^{*} \cdot \chi_{s}^{a}\right|_{\omega=\omega_{r}} \cdot \boldsymbol{E} / 4 W,
$$

Table 1

Values of Fixed Plasma Parameters in Our Study

\begin{tabular}{lc}
\hline \hline Quantity & Value \\
\hline Proton plasma beta $\beta_{p}$ & 0.4 \\
Temperature ratio $T_{c} / T_{p}$ & 2 \\
$T_{h} / T_{p}$ & 10 \\
Density ratio $n_{c} / n_{h}$ & 9 \\
Proton bulk drift $v_{p} / v_{\mathrm{A}}$ & 0 \\
Angle between $\boldsymbol{k}, \boldsymbol{B}_{0}$ & $89^{\circ}$ or $91^{\circ}$ \\
\hline
\end{tabular}

where $\chi_{s}^{a} \equiv\left(\chi_{s}-\chi_{s}^{\dagger}\right) / 2 i$ is the anti-Hermitian part of $\chi_{s}$, $\omega_{r} \equiv \mathfrak{R}(\omega)$ and $W$ is the wave energy.

In the weak damping limit, $P_{s}$ gives the fraction of wave energy damped by plasma component $s$ during one wave period. The total heating rate $P_{\text {total }} \equiv \sum_{s} P_{s}$ corresponds to the wave energy dissipation rate directly calculated from the damping rate, i.e., $1-e^{2 \omega_{i} T}$, where $\omega_{i} \equiv \Im(\omega)$ and $T \equiv 2 \pi / \omega_{r}$ is the wave period, as long as $\omega_{i}$ is small compared to $\omega_{r}$. In this letter, we focus on the linear damping of KAWs. Electron drifts may, however, alter wave-particle interactions to such a degree that the corresponding waves become unstable, which we note for the sake of completeness. Notice that $P_{s}$ may become negative, corresponding to cooling of species $s$.

\section{RESULTS}

Figures 2(a) and (b) show dispersion relations of KAWs in plasmas with $\delta v_{c} \in[-4,4]$. Panel (a) demonstrates that electron drifts have little effect on the real part of the KAW frequencies, consistent with Gary et al. (1975). Indeed, the protons rather than the electrons mainly determine the dynamics of low-frequency KAWs. Panel (b) compares damping rates of KAWs in plasmas with different electron drifts. Negative (positive) $\delta v_{c}$ lead to an enhancement (reduction) of the KAW damping at all wavenumbers. Panel (b) shows that $\delta v_{c} \sim-4$ leads to an increase in the damping rate by $\sim 50 \%$ compared to the case without electron drifts. For sufficiently large positive $\delta v_{c}$, KAWs become unstable in certain wavenumber ranges. This instability is an example of a "heat flux instability" (Gary et al. 1975). The two-population electrons in our model introduce an electron heat flux (third moment of the electron VDF), which may provide energy to drive certain wave modes (e.g., whistler, Alfvén, and magnetosonic) unstable (Gary et al. 1975, 1998a, 1999). These heat flux instabilities, in return, regulate the electron heat flux and hence the electron drifts. We note that there is no conflict between the damping and growth of KAWs and other wave modes. For instance, parallel whistler waves, whose instability threshold is much lower than that of the Alfvénic instability, are unstable even if $\delta v_{c}>-4$. Therefore, the Alfvénic instability cannot regulate the electron heat flux in the solar wind and is more of academic interest under typical solar wind conditions since the instability with the lowest threshold constrains the electron heat flux once triggered. Figure 2(c) compares the total particle heating rate (solid) with the wave energy dissipation rate (dotted). When $1-e^{2 \omega_{i} T} \lesssim 0.5$, i.e., the wave retains more than half of its energy after a wave period, $P_{\text {total }} \approx 1-e^{2 \omega_{i} T}$. Hence we show that $P_{s}$ is a good measure of energy flow as long as damping is weak. 

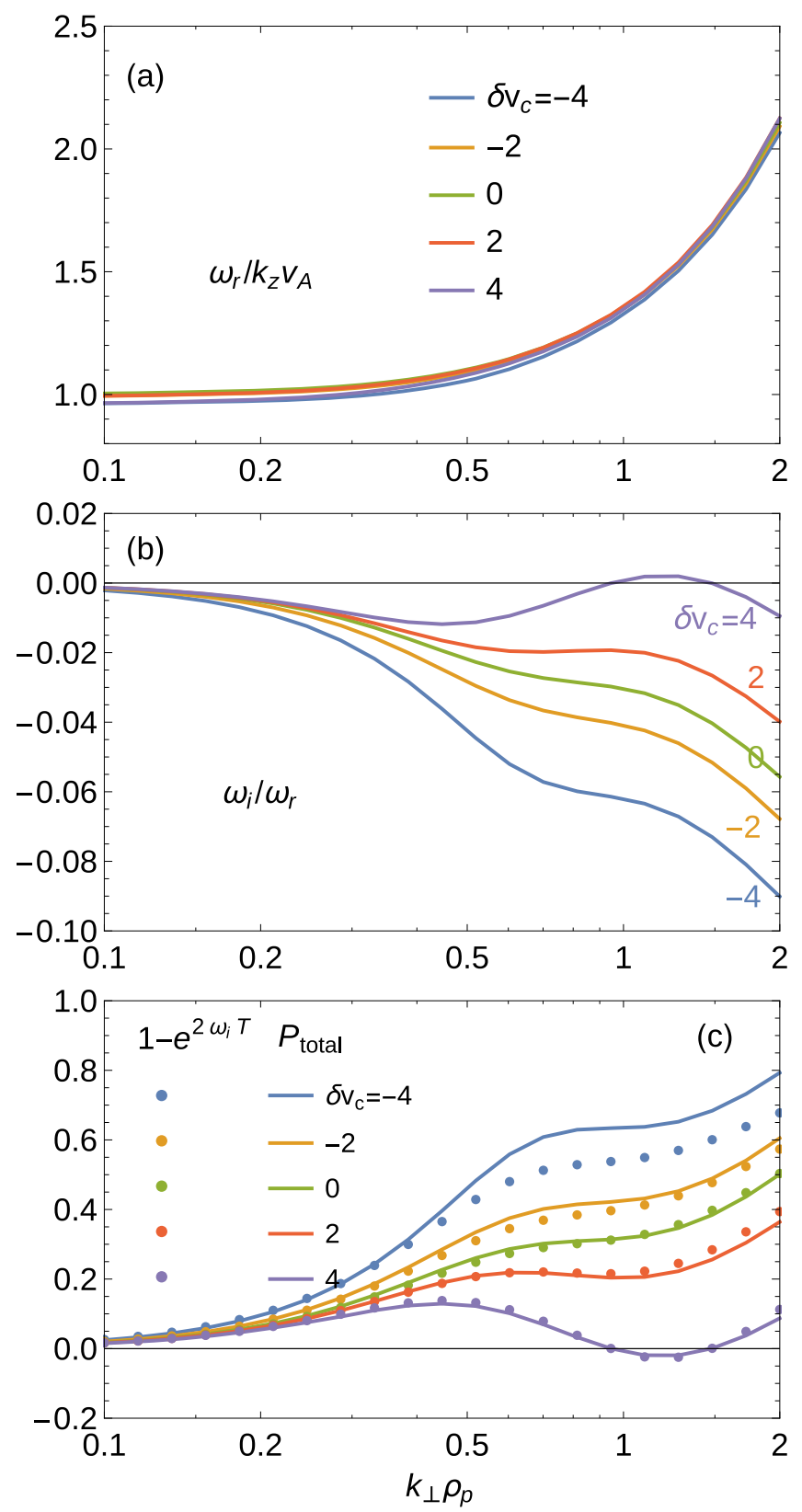

Figure 2. (a)-(b) Dispersion relation of KAWs in plasmas for different $\delta v_{c}$. $\omega_{r}$ and $\omega_{i}$ are the real and imaginary parts of the wave frequency. $\rho_{p} \equiv v_{T p} / \Omega_{p}$ is the proton gyro-radius, $v_{T p} \equiv \sqrt{2 k_{B} T_{p} / m_{p}}$ is the proton thermal speed, and $\Omega_{p} \equiv e B_{0} / m_{p} c$ is the proton gyro-frequency. (c) Total particle heating rates $\left(P_{\text {total }}\right.$, solid lines $)$ and wave energy dissipation rates $\left(1-e^{2 \omega_{i} T}\right.$, dots $)$ as functions of wavenumber.

Figures 3(a)-(d) present heating rates for protons, all electrons (core + halo), as well as separately for core electrons and halo electrons. Recall that positive $P_{s}$ indicates heating for species $s$, and that negative $P_{s}$ indicates cooling. Panel (a) shows that protons are always heated, although electron drifts can slightly modify the values of the proton heating rate. On the other hand, panels (b)-(d) show that an electron population can experience both cooling and heating depending on $\delta v_{c}$. While both core and halo are heated in the absence of bulk drifts, sufficiently large positive $\delta v_{c}$ lead to core cooling and at the same time significantly enhance halo heating. Negative $\delta v_{c}$ have the opposite effect on electron heating. In general, electron drifts have a larger effect on electron heating than on proton heating. Electron drifts modify the damping rates of KAWs by changing the efficiency of wave-particle interactions for the different electron populations.

Figure 4 shows how the energy partition depends on electron drifts at $k_{\perp} \rho_{p}=1 . P_{c} / P_{p}$ and $P_{h} / P_{p}$ vary wildly with $\delta v_{c}$. However, since electron drifts affect core heating and halo heating in opposite ways, the total electron heating and consequently the electron-proton energy partition reveal a more moderate dependence on electron drifts. Nevertheless, a core drift of $\delta v_{c} \sim-4$ increases $P_{e} / P_{p}$ by $\sim 25 \%$. Without electron drifts, electron heating dominates over proton heating in hydrogen plasmas at $k_{\perp} \rho_{p} \sim 1$ for $\beta \sim 1$ (Quataert 1998). We see in Figure 4 that positive $\delta v_{c}$ significantly reduces electron-to-proton heating ratio. At $\delta v_{c} \sim 3$, electron heating becomes negligible compared to proton heating. We note at this point that the dependence of energy partition on electron drifts is qualitatively the same at other wavenumbers, which can be inferred from Figure 3.

We interpret the dependence of wave damping and particle heating on electron drifts in terms of a simplified resonance analysis. Since $\omega_{r}$ is almost independent of electron drifts, the proton heating rate shows only a small variation. However, core and halo electron bulk drifts significantly change the value and the gradient of the electron VDF at the resonance speed,

$$
v_{\mathrm{res}} \equiv \frac{\omega_{r}-n \Omega_{e}}{k_{\|}},
$$

where $\Omega_{e}$ is the electron gyro-frequency and $n$ is an integer depending on the polarization properties of the wave mode (Marsch 2006).

In our case, the most relevant resonance for wave-particle interactions with KAWs is the Landau resonance with $n=0$. Electrons with the (field-parallel) speed $v_{\text {res }}$ interact resonantly with the corresponding wave mode. We illustrate the case for negative $\delta v_{c}$ schematically in Figure 1.

The gradient of the distribution functions at the resonance speed determines if the particle species gains or loses energy, i.e., the signs of $P_{p}, P_{c}$, and $P_{h}$. In the example shown in Figure 1, the resonance occurs where the halo VDF has a positive gradient, and hence the KAW removes energy from the halo electrons, leading to a negative value for $P_{h}$. In contrast, core electrons and protons have resonances where the gradients of their VDFs are negative, leading to positive values for $P_{c}$ and $P_{p}$. Similarly, the dependence of $P_{s}$ on $\delta v_{c}$ can also be inferred from the positions of the resonance points with respect to the VDFs. However, a qualitative resonance analysis cannot provide a thorough comparison across the plasma components. We find that $P_{s}$, defined in Equation (2), gives a better understanding of the energy transfer between waves and different plasma components.

It is worth noting that electron drifts seem to provide a collisionless energy coupling between core and halo electrons (see the case with $\delta v_{c}=-2$ for instance). Figure 2(c) shows that $1-e^{2 \omega_{i} T} \approx P_{c}+P_{h}+P_{p}$. With $P_{c}, P_{p}>0$ and $P_{h}<0$ (from Figure 3$),\left(1-e^{2 \omega_{i} T}\right)+\left(-P_{h}\right) \approx P_{c}+P_{p}$, meaning that energy flows from damped waves and halo electrons into core electrons and protons. A similar energy transport among electron components was suggested by Gary et al. (1998b). According to this study, the Alfvénic heat-flux instability transfers energy from the drifting halo into the electron core 

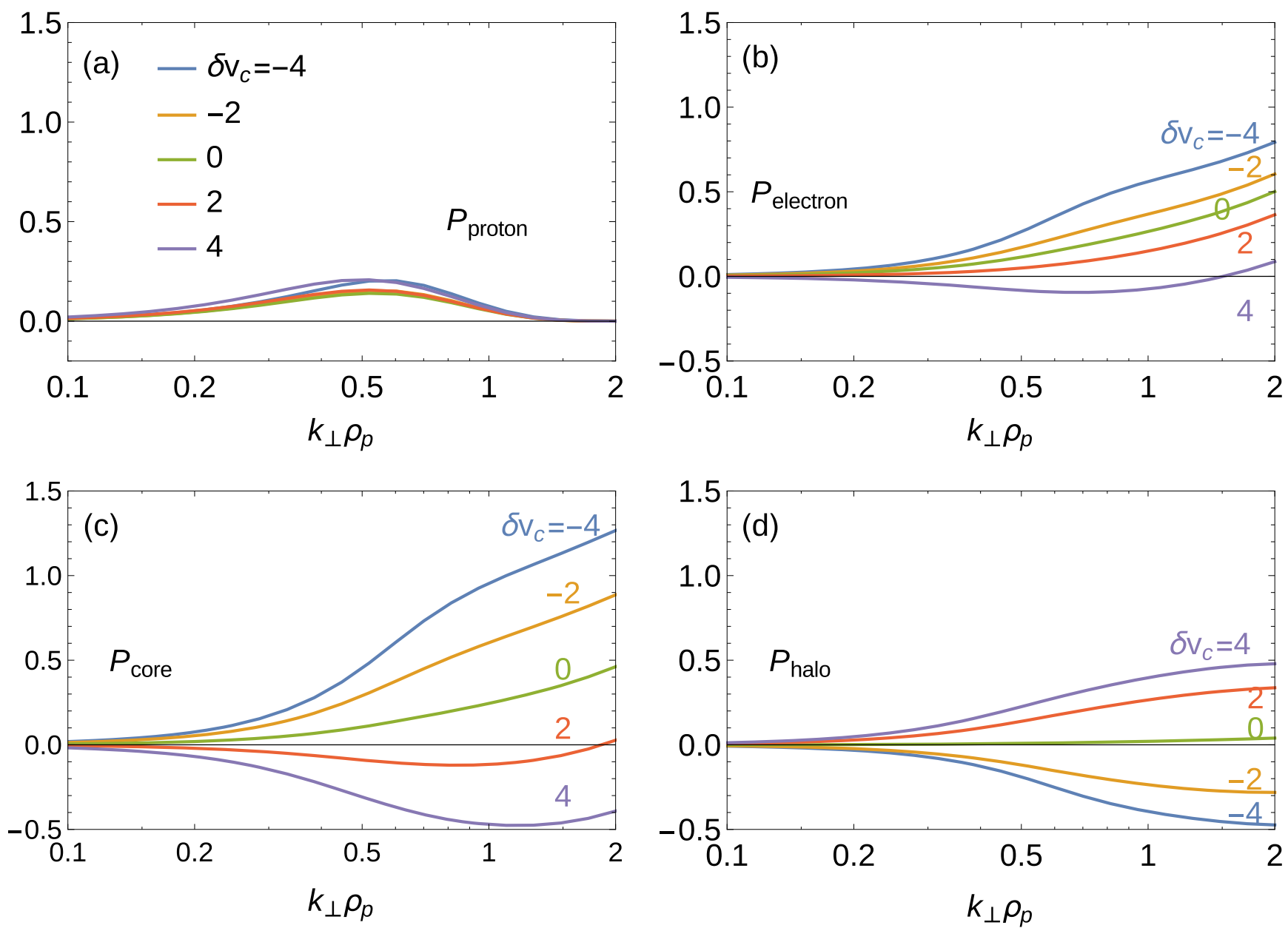

Figure 3. Dependence of $P_{\text {proton }}(\mathrm{a}), P_{\text {electron }}(\mathrm{b}), P_{\text {core }}(\mathrm{c})$, and $P_{\text {halo }}(\mathrm{d})$ on $k_{\perp} \rho_{p}$ in plasmas with varying $\delta v_{c}$.

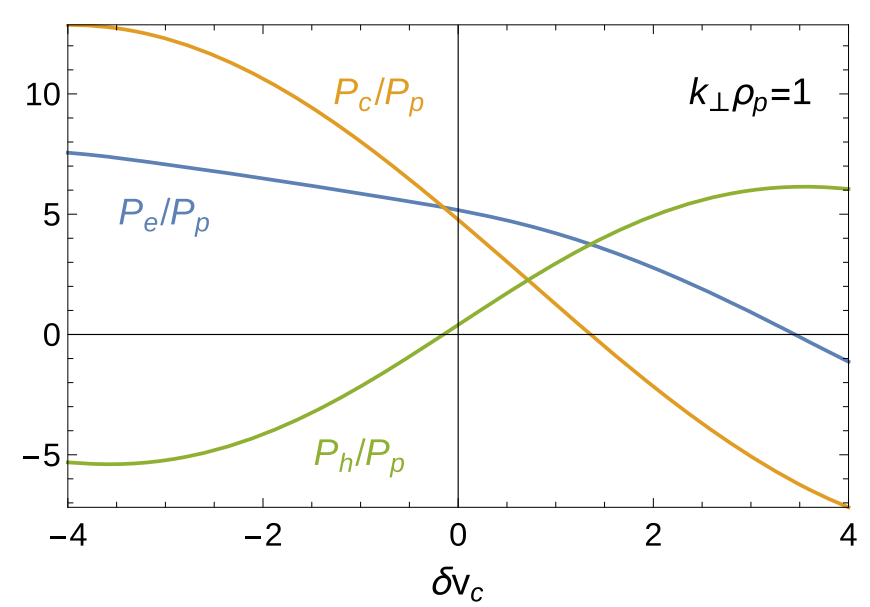

Figure 4. Ratio of energy partition between electrons and protons as a function of electron drift.

through the Landau-resonant absorption of the waves, leading to a lower limit for $\beta_{c}$.

\section{DISCUSSION AND CONCLUSION}

For the sake of clarity, we present results for a single propagation angle in this letter. A careful analysis shows that other oblique angles do not lead to any qualitative differences. Given a fixed KAW power spectrum, we anticipate that the presence of electron drifts changes the wave-energy dissipation rate, particle heating rates, and the proton-electron energy partition ratio significantly.

In the context of the solar wind, since core electrons are nearly always observed to drift sunward in the proton frame, positive (negative) $\delta v_{c}$ correspond to KAWs propagating sunward (anti-sunward). Therefore, anti-sunward KAWs experience stronger damping in the presence of electron drifts and lead to a stronger core heating, halo electron cooling, and a stronger total electron heating. Since the total energy flux typically points anti-sunward in the solar wind, we suggest that electron drifts lead to stronger electron heating than expected from previous calculations (Quataert 1998; Leamon et al. 1999). An interesting corollary of this work is that since the core electron drift speed depends on the collisionality of the solar wind (Pulupa et al. 2014), the heating rate as calculated here may therfore also indirectly depend on solar wind Couloumb collisions.

Our model includes relative drifts in the electron VDF, while it ignores several additional features of solar wind electrons, namely the presence of superthermal electrons, one-sided beams (strahl) and temperature anisotropies. By using a Maxwellian halo, we ignore high energy tails in the electron VDF. However, this assumption does not significantly affect the gradients of the electron distribution function at $v_{\text {res }} \sim v_{\mathrm{A}}$ which mainly determine the damping. We can apply a similar argument to strahl electrons, which also occupy a different 
region in the velocity space. Regarding temperature anisotropies, Gary et al. (1975) studied the dependence of the core drift threshold for the Alfvénic instability on electron temperature anisotropy and found that the dependence is weak in plasmas with $\beta_{p}<0.25$. We expect a similar weak relation in our case, since Landau-resonant instabilities show such a weak dependence in general (Verscharen \& Chandran 2013), since the strength of this type of resonant interaction is determined by the parallel gradients of the electron distribution function. If the parallel temperature is kept constant, the introduction of temperature anisotropy only changes the perpendicular gradients of the VDF and therefore does not significantly alter the wave-particle interaction.

In this letter, we choose a fiducial set of representative solar wind plasma parameters to demonstrate the effects of electron drifts on the damping of KAWs. In order to fully account for all of the relevant effects in the solar wind, it is important to conduct a full scan of the corresponding parameter space. This endeavor is beyond the scope of this work and will be presented in a future paper.

Our work shows that, despite our limiting assumption of a superposition of Maxwellians to represent the electron distribution function, its fine-structure has a strong influence on the propagation and damping properties of KAWs. Therefore, our work is of relevance in the broader context of all collisionless astrophysical plasmas in which non-Maxwellian electron distributions can develop and persist. Considering that most astrophysical plasmas are in a turbulent state and that $\mathrm{KAW}$-turbulence is believed to be the dominant type of plasma turbulence on small scales, our work suggests that the effects of non-Maxwellian electron distributions be carefully accounted for in studies of collisionless astrophysical plasmas in general.

We are grateful to Christopher C. Chaston, Marc P. Pulupa, Eliot Quataert, and Kristopher G. Klein for helpful discussions. Y.T. is supported by NASA grant APL-975268 and Charles K. Kao Scholarship. S.D.B. is supported by NASA grant APL975268. C.H.K.C. is supported by an Imperial College Junior Research Fellowship. C.S. is supported by NASA grant
NNX14AC07G. D.V. is supported by NASA grant NNX12AB27G.

\section{REFERENCES}

Alexandrova, O., Chen, C. H. K., Sorriso-Valvo, L., Horbury, T. S., \& Bale, S. D. 2013, SSRv, 178, 101

Bale, S. D., Kellogg, P. J., Mozer, F. S., Horbury, T. S., \& Reme, H. 2005, PhRvL, 94, 215002

Bale, S. D., Pulupa, M., Salem, C., Chen, C. H. K., \& Quataert, E. 2013, ApJL, 769, L22

Belcher, J. W., \& Davis, L., Jr. 1971, JGR, 76, 3534

Boldyrev, S., \& Perez, J. C. 2012, ApJL, 758, L44

Bruno, R., \& Carbone, V. 2013, LRSP, 10, 2

Chandran, B. D. G., Verscharen, D., Quataert, E., et al. 2013, ApJ, 776, 45

Chen, C. H. K., Boldyrev, S., Xia, Q., \& Perez, J. C. 2013, PhRvL, 110, 225002

Chen, C. H. K., Horbury, T. S., Schekochihin, A. A., et al. 2010, PhRvL, 104, 255002

Cranmer, S. R., \& van Ballegooijen, A. A. 2003, ApJ, 594, 573

Feldman, W. C., Asbridge, J. R., Bame, S. J., Montgomery, M. D., \& Gary, S. P. 1975, JGR, 80, 4181

Gary, S. P., Feldman, W. C., Forslund, D. W., \& Montgomery, M. D. 1975, JGR, 80, 4197

Gary, S. P., Li, H., O’Rourke, S., \& Winske, D. 1998a, JGR, 103, 14567

Gary, S. P., Newbury, J. A., \& Goldstein, B. E. 1998b, JGR, 103, 14559

Gary, S. P., Skoug, R. M., \& Daughton, W. 1999, PhPl, 6, 2607

Goldreich, P., \& Sridhar, S. 1995, ApJ, 438, 763

Horbury, T. S., Forman, M., \& Oughton, S. 2008, PhRvL, 101, 175005

Howes, G. G., Klein, K. G., \& TenBarge, J. M. 2014, arXiv:1404.2913

Howes, G. G., Tenbarge, J. M., Dorland, W., et al. 2011, PhRvL, 107, 035004

Kasper, J. C., Maruca, B. A., Stevens, M. L., \& Zaslavsky, A. 2013, PhRvL, 110,091102

Leamon, R. J., Smith, C. W., Ness, N. F., \& Wong, H. K. 1999, JGR, 104, 22331

Maksimovic, M., Zouganelis, I., Chaufray, J.-Y., et al. 2005, JGR, 110, 9104 Marsch, E. 2006, LRSP, 3, 1

Pulupa, M. P., Bale, S. D., Salem, C., \& Horaites, K. 2014, JGR, 119, 647

Quataert, E. 1998, ApJ, 500, 978

Sahraoui, F., Goldstein, M. L., Belmont, G., Canu, P., \& Rezeau, L. 2010, PhRvL, 105, 131101

Salem, C. S., Howes, G. G., Sundkvist, D., et al. 2012, ApJL, 745, L9

Schekochihin, A. A., Cowley, S. C., Dorland, W., et al. 2009, ApJS, 182, 310

Stix, T. H. 1992, Waves in Plasmas (New York: AIP)

Swanson, D. G. 1989, Plasma Waves (New York: Academic)

Tu, C.-Y., \& Marsch, E. 1995, SSRv, 73, 1

Verscharen, D., \& Chandran, B. D. G. 2013, ApJ, 764, 88 